\title{
Application of homotopy perturbation method for MHD boundary layer flow of an upper-convected Maxwell fluid in a porous medium
}

\author{
Anuj Kumar Jhankal
}

${ }^{l}$ Department of Mathematics, Birla Institute of Technology, Mesra, Ranchi, Jaipur Campus, Jaipur-302 017, India. Article Info: Submitted on December 22, 2014, Accepted on July 20, 2015.

\begin{abstract}
The magneto-hydrodynamics (MHD) boundary layer flow of an electrically conducting upper-convected Maxwell (UCM) fluid in porous medium is studied. The governing similarity equation is solved by He's Homotopy perturbation method (HPM). The main advantage of HPM is that it does not require the small parameters in the equations and hence the limitations of traditional perturbation can be eliminated. The results reveal that the proposed method is very effective and simple and can be applied to other nonlinear problems. The effects of various physical parameters on the flow presented and discussed through graphs.
\end{abstract}

Keywords: MHD; Boundary layer flow; Upper-convected Maxwell fluid; Homotopy perturbation method; Porous medium.

\section{Introduction}

In recent years, the studies of boundary layer flows of non-Newtonian fluid have received considerable attention due to their numerous industrial and engineering applications. The non-Newtonian fluids in view of their diverse rheological properties cannot be examined through one constitutive relationship between shear stress and rate of strain. Many models of non-Newtonian fluids exist. Maxwell model has become the most popular as it can predict stress relaxation and also excludes the complicating effects of shear dependent viscosity. In view of its simplicity, this fluid model has acquired special status amongst model has acquired special status amongst the recent workers in the field.

The MHD phenomenon is characterized by an interaction between the hydrodynamic and boundary layer electromagnetic field. The earliest investigation in this area was conducted by Alfvén around 1940. Most of the main applications of MHD in nature are the Earth's core and solar flares, and also it has many applications in engineering such as the electromagnetic casting of metals and the confinement of plasmas Gurion. ${ }^{1}$

Sarpakaya $^{2}$ was the first researcher to study the MHD flow of non-Newtonian fluid. UCM fluid has been the topic of several research Renardy, ${ }^{3}$ Rao and Rajagopal, ${ }^{4}$ Qi and $\mathrm{Xu},{ }^{5}$ Hayat and Sajid, ${ }^{6}$ Hayat et al., ${ }^{7}$ Jamil et al., ${ }^{8}$ Salah et al. ${ }^{9}$ and Hayat et al. ${ }^{10}$ Wang and $\operatorname{Tan}^{11}$ discussed the flow of Maxwell fluid in a porous medium. Zierep and Fetecau ${ }^{12}$ studied Rayleigh-Stokes problem using Maxwell fluid and find exact solution. Sadeghy et al. ${ }^{13}$ did a comparative study for Sakiadis flow of an upper-convected Maxwell fluid on a rigid plate.

The governing equations of non-Newtonian fluids are very complicated and highly nonlinear as compared to those for Newtonian fluids. There are few analytical solutions of the equations involving Newtonian fluids and such solutions become even rarer when equations for non-Newtonian fluids are considered. In this paper one of the semi-exact method which is called He's Homotopy Perturbation Method (HPM) has been introduced and its application MHD boundary layer flow of an upper-convected Maxwell in a porous medium. The initial work in HPM was studied by J. H. $\mathrm{He}^{14-18}$ and after that these investigations inspired a lot of researchers Ariel et al., ${ }^{19}$ Beléndez et al., ${ }^{20}$ Ganji and Rajabi, ${ }^{21}$ Ganji and Ganji, ${ }^{22}$ Hosein et al., ${ }^{23}$ Jhankal, ${ }^{24}$ Ma et al., ${ }^{25}$ Siddiqui et al., ${ }^{26}$ Zhang and $\mathrm{He}^{27}$ Zhang et al., ${ }^{28}$ and many others to solve nonlinear equations with this method.

Basic idea of homotopy perturbation method (HPM):

To illustrate the basic ideas of the HPM, we consider the following nonlinear differential equation.

$\mathrm{A}(\mathrm{u})-\mathrm{f}(\mathrm{r})=0, \mathrm{r} \in \Omega$

Subject to the boundary conditions

$B\left(u, \frac{\partial u}{\partial \eta}\right)=0, r \in \Gamma$

where $\mathrm{A}$ is a general differential operator, $\mathrm{B}$ is a boundary operator, $f(r)$ is a known analytical function and $\Gamma$ is the boundary of the domain $\Omega$. A can be divided into two parts which are $\mathrm{L}$ and $\mathrm{N}$, where $\mathrm{L}$ is 


\section{Chemical Engineering Research Bulletin 18(2015) 12-17}

linear and $\mathrm{N}$ is nonlinear. Therefore eqn. 1 can be rewritten as follows:

$\mathrm{L}(u)+N(u)-f(r)=0, r \in \Omega$

By the homotopy perturbation technique, we construct a homotopy

$v(r, p): \Omega \times[0,1] \rightarrow R$, which satisfies:

$H(v, p)=(1-p)\left[L(v)-L\left(u_{0}\right)\right]+p[A(v)-f(r)]=$

$0, p \in[0,1], r \in \Omega$

where $p \in[0,1]$ is an embedding parameter and $u_{-} 0$ is an initial approximation that satisfies the boundary condition. Obviously, from these definitions we will have: $\quad H(v, 0)=L(v)-L\left(u_{0}\right)=0$

$$
H(v, 1)=A(v)-f(r)=0
$$

The changing process of $\mathrm{p}$ from zero to one is just that of $v(r, p)$ from $u_{-} 0(r)$ to $u(r)$. In topology, this is called deformation and $\mathrm{L}(\mathrm{v})-\mathrm{L}\left(\mathrm{u}_{-} 0\right)$ and $\mathrm{A}(\mathrm{v})-\mathrm{f}(\mathrm{r})$ are called homotopy. According to the HPM, we can first use the embedding parameter $p$ as a "small parameter" and assuming that the solution of (4) can be written as a power series in $\mathrm{p}$ :

$v=v_{0}+p v_{1}+p^{2} v_{2} \ldots$

Setting $\mathrm{p}=1$, results in the approximate solution of (1):

$u=\lim _{p \rightarrow 1} v=v_{0}+v_{1}+v_{2}+\cdots$

The convergence and stability of this method was shown in Hosein et al. ${ }^{29}$

\section{Materials and Methods}

\section{Formulation of the problem}

Consider the steady and incompressible MHD boundary layer flow an electrically conducting fluid obeying UCM model in a porous medium. The $\mathrm{x}$ - and $\mathrm{y}$-axes are taken along and perpendicular to the flow, respectively. A uniform magnetic field of strength B_0 is imposed along the $y$-axis. The induced magnetic field is negligible, which is a valid assumption on a laboratory scale under the assumption of small magnetic Reynolds number and the external electric field is zero. Under the usual boundary layer approximations, the governing equation of continuity and momentum $\left(\mathrm{Pai}^{30}\right.$ Schlichting, ${ }^{31}$ under the influence of externally imposed transverse magnetic field are:

$\frac{\partial u}{\partial x}+\frac{\partial u}{\partial y}=0$

$u \frac{\partial u}{\partial x}+v \frac{\partial u}{\partial y}+\lambda\left(\mathrm{u}^{2} \frac{\partial^{2} \mathrm{u}}{\partial x^{2}}+2 \mathrm{uv} \frac{\partial^{2} \mathrm{u}}{\partial x \partial y}+\mathrm{v}^{2} \frac{\partial^{2} \mathrm{u}}{\partial y^{2}}\right)=v \frac{\partial^{2} u}{\partial x^{2}}-$ $\frac{\sigma_{e} B_{0}^{2}}{\rho} u-\frac{v u}{K}$

Along with the boundary conditions for the problem are given by:

$u(x, 0)=U, v(x, 0)=0$,

$u(x, \infty) \rightarrow 0$

The continuity eqn. 7 is satisfied by introducing a stream function $\Psi$ such that $\mathrm{u}=\partial \Psi / \partial \mathrm{y}$ and $=-\partial \Psi / \partial \mathrm{x}$
The momentum equation can be transformed into the corresponding ordinary nonlinear differential equation by the following transformation:

$\eta=y \sqrt{\frac{U}{v x}}, \Psi=\sqrt{v x U} f(\eta)$

where $\eta$ is the independent similarity variable. The transformed nonlinear ordinary equation is

$2 f^{\prime \prime \prime}-\beta\left(2 f f^{\prime} f^{\prime \prime}+f^{2} f^{\prime \prime \prime}+\eta f^{\prime 2} f^{\prime \prime}\right)+f f^{\prime \prime}-2(M+$

$S) f^{\prime}=0$

The transformed boundary conditions are:

$f(0)=0, f^{\prime}(0)=1, f^{\prime}(\infty) \rightarrow 1$

where prime denotes differentiation with respect to $\eta$, $\mathrm{M}=\left(\sigma \_\right.$e $\left.\mathrm{B} \_0^{\wedge} 2\right) / \rho \quad \mathrm{x} / \mathrm{U}$ is the dimensionless magnetic parameter and $S=v x / K U$ is the porosity parameter.

\section{Solution with Homotopy perturbation method}

According to the HPM, the homotopy form of eqn. 11 is constructed as follows:

$(1-p)\left[2 f^{\prime \prime \prime}-2(M+S) f^{\prime}\right]+p\left[2 f^{\prime \prime \prime}-\beta\left(2 f f^{\prime} f^{\prime \prime}+\right.\right.$ $\left.\left.f^{2} f^{\prime \prime \prime}+\eta f^{\prime 2} f^{\prime \prime}\right)+f f^{\prime \prime}-2(M+S) f^{\prime}\right]=0$

We consider $f$ as:

$f=f_{0}+p f_{1}+p^{2} f_{2} \cdots$

By substituting eqn. 14 into 13, and then

(i) Terms independent of $\mathrm{p}$ give

$2 f_{0}^{\prime \prime \prime}-2(M+S) f_{0}^{\prime}=0$

The boundary conditions are

$f_{0}(0)=0, f_{0}^{\prime}(0)=1, f_{0}^{\prime}(\infty)=1$.

(ii) Terms containing only $\mathrm{p}$ give

$2 f_{1}^{\prime \prime \prime}-\beta\left(2 f_{0} f_{0}^{\prime} f_{0}^{\prime \prime}+f_{0}^{2} f_{0}^{\prime \prime \prime}+\eta f_{0}^{\prime 2} f_{0}^{\prime \prime}\right)+f_{0} f_{0}^{\prime \prime}-$

$2(M+S) f_{1}^{\prime}=0$

The boundary conditions are

$f_{1}(0)=0, f_{1}^{\prime}(0)=0, f_{1}^{\prime}(\infty)=0$.

Solving eqns. 15 and 17 with boundary conditions (16) and (18) respectively, we have

$f_{0}=\frac{A_{1}-e^{\sqrt{(M+S) \eta}} e^{-5 \sqrt{(M+S)}}+e^{-\sqrt{(M+S) \eta}} e^{5 \sqrt{(M+S)}}}{A_{2}}$

$f_{1}=A_{17}+A_{18} e^{\sqrt{(M+S) \eta}}+A_{19} e^{-\sqrt{(M+S) \eta}}+$ $A_{20} \eta e^{\sqrt{(M+S)} \eta}+A_{21} \eta e^{-\sqrt{(M+S)} \eta}+A_{22} \eta^{2} e^{\sqrt{(M+S)} \eta}+$ $A_{23} \eta^{2} e^{-\sqrt{(M+S)} \eta}+A_{24} e^{2 \sqrt{(M+S)} \eta}+A_{25} e^{-2 \sqrt{(M+S) \eta}}+$ $A_{26} e^{3 \sqrt{(M+S)} \eta}+A_{27} e^{-3 \sqrt{(M+S)} \eta}+A_{28} \eta e^{3 \sqrt{(M+S)} \eta}+$ $A_{29} \eta e^{-3 \sqrt{(M+S)} \eta}+A_{30} \eta$

The constant coefficients, can be calculated using boundary conditions, the boundary condition $\eta=\infty$ were replaced by those at $\eta=5$ in accordance with 


\section{Chemical Engineering Research Bulletin 18(2015) 12-17}

standard practice in the boundary layer analysis. If $\mathrm{p} \rightarrow 1$, we find the approximate solution of equation (11). The constant coefficients A_i $(i=1,2,3, \ldots .30)$ are defined as usually.

\section{Results and Discussion}

The governing eqn.11 is solved subject to boundary condition (12) using Homotopy perturbation method (HPM). In this section, we present the result showing velocity distribution and skin-friction at the surface. In order to show the important features of the flow, the governing equation is solved for the selected values of the governing parameters $M, \beta$ and $S$, and the results are illustrated graphically in Figs. 1-7.

Figs. 1 and 2 show the effect of magnetic parameter $M$ for the given values of Deborah number $\beta$ and porosity parameter S. An increase in the M leads in decrease of both the velocity components $f(\eta)$ and $f^{\wedge^{\prime}}(\eta)$ and thereby decreases the thickness of the boundary layer. This is due to the fact that applied transverse magnetic field presents a damping effect on the flow velocity by creating a drag force that opposes motion, which thereby causes the velocity to decrease.

Figs. 3 and 4 show the effect of Deborah number $\beta$ for the given values of magnetic parameter $\mathrm{M}$ and porosity parameter S. It is observed that the both the velocity components $f(\eta)$ and $f^{\wedge^{\prime}}(\eta)$ increases and thereby increases the thickness of the boundary layer with an increase in $\beta$. Since the Deborah number $\beta$ is dependent upon $\lambda$ (retardation time), physically larges retardation time of any material makes it less viscous resulting in an increase in its motion.

Figs. 5 and 6 show that the velocity components $f(\eta)$ and $\mathrm{f}^{\wedge \prime}(\eta)$ decreases due to increase in porosity parameter $\mathrm{S}$ which agrees with natural phenomena.

Fig. 7 represents the skin friction parameter against magnetic parameter $M$ for various values of Deborah number $\beta$. It is noted that the effect of increasing $M$ is to decrease the skin friction at the surface. The positive values of $\mathrm{f}^{\wedge}$ " $(0)$ imply that fluid exerts the drag force on the surface, and the negative values $\mathrm{f}^{\wedge}$ " $(0)$ imply the opposite meaning. Thus as $\mathrm{M}$ becomes very large, there will be a decrease in skin friction $\mathrm{f}^{\wedge \prime}(0)$, and then a decrease in the drag force at the surface. Figure also shows that an increase in the Deborah number $\beta$ leads to increases the skin friction $\mathrm{f}^{\wedge \prime}(0)$ and the drag force at the surface.

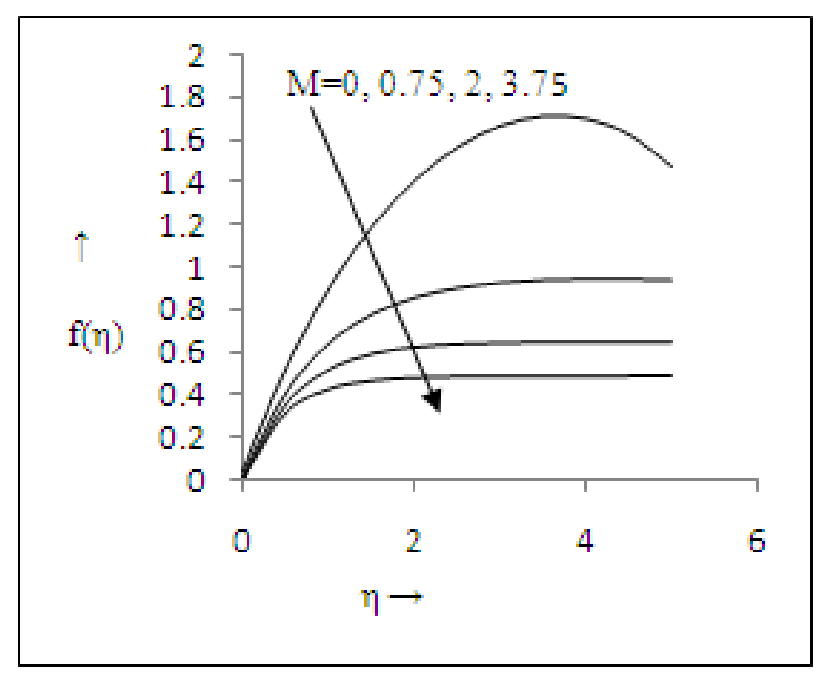

Figure 1: Effects of magnetic parameter $M$ on $f(\eta)$, when $\beta=0.5$ and $S=0.25$.

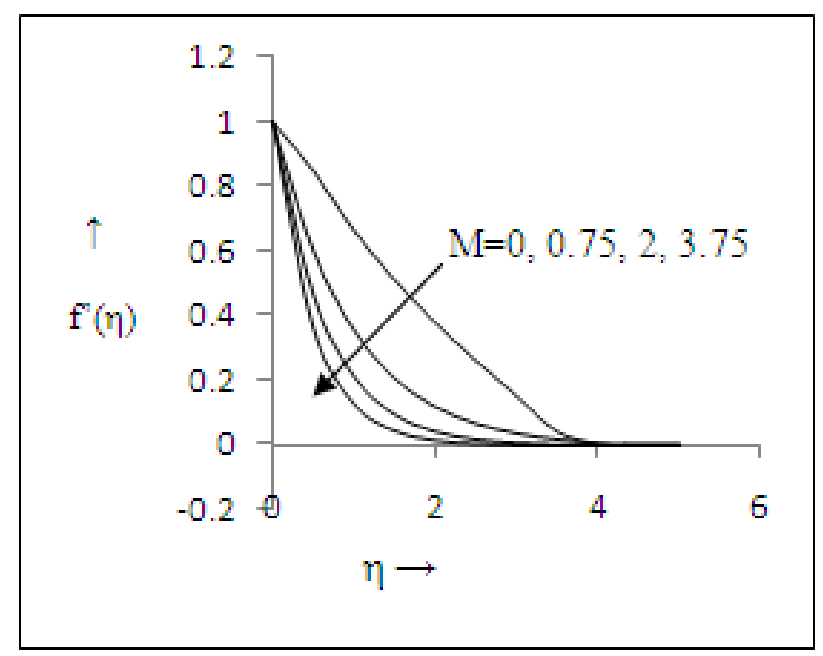

Figure 2: Effects of magnetic parameter $M$ on $f^{\wedge \prime}(\eta)$, when $\beta=0.5$ and $S=0.25$.

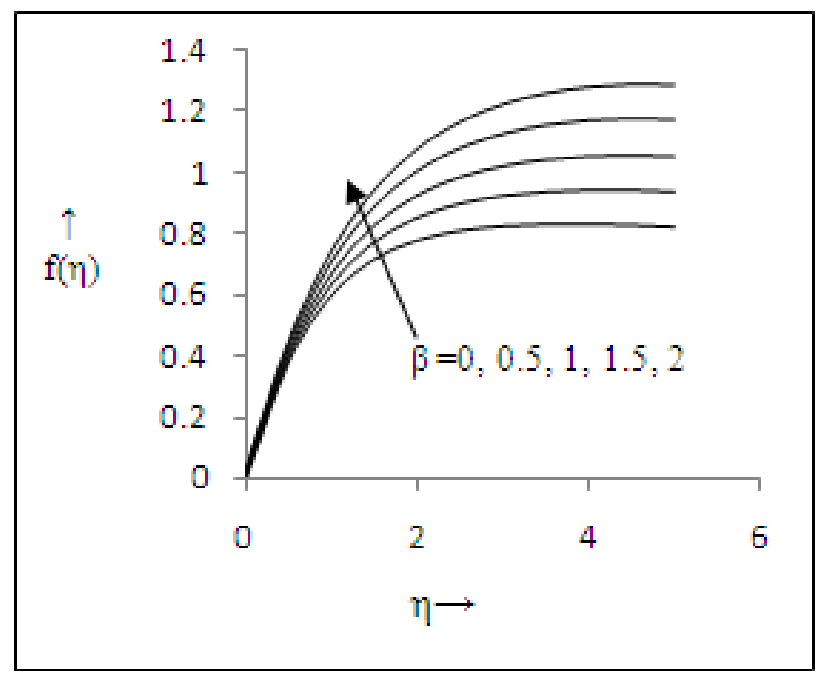

Figure 3: Effects of Deborah number $\beta$ on $\mathrm{f}(\eta)$, when $\mathrm{M}=0.5$ and $\mathrm{S}=0.5$. 


\section{Chemical Engineering Research Bulletin 18(2015) 12-17}

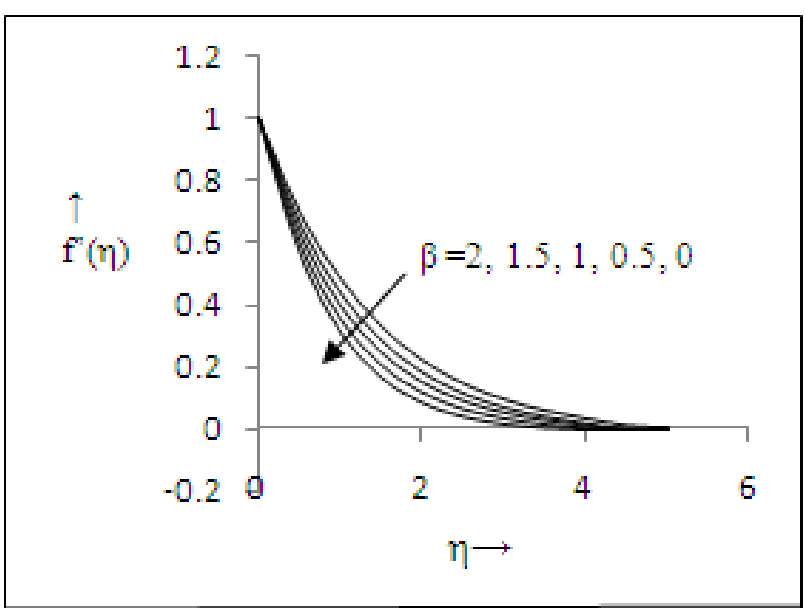

Figure 4: Effects of Deborah number $\beta$ on $\mathrm{f}^{\wedge^{\prime}}(\eta)$, when $\mathrm{M}=0.5$ and $\mathrm{S}=0.5$.

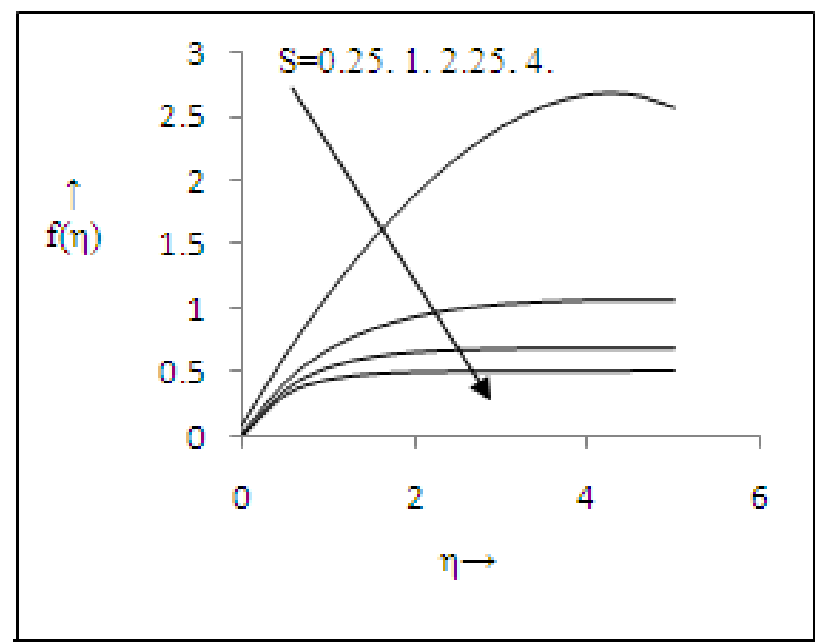

Figure 5: Effects of the porosity parameter $S$ on $f(\eta)$, when $\mathrm{M}=0.0$ and $\beta=1.0$.

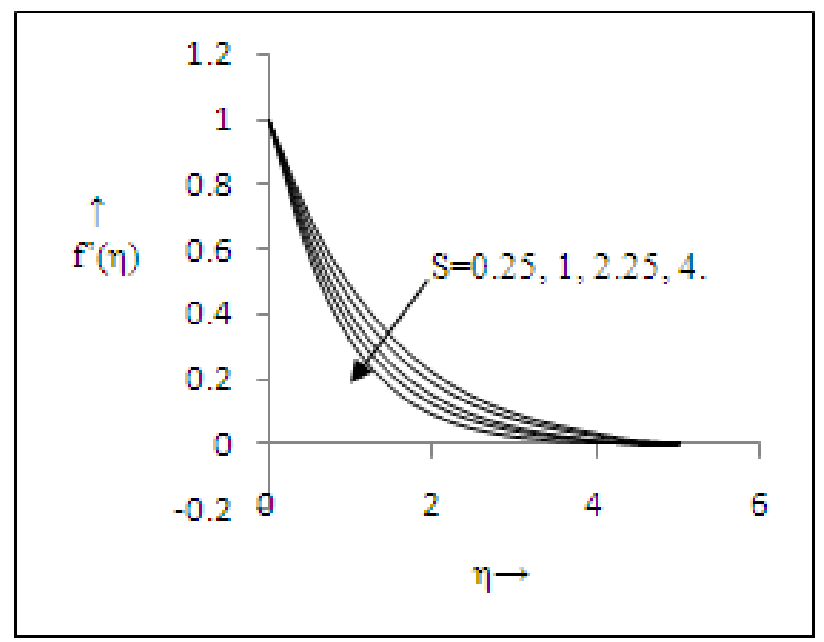

Figure 6: Effects of the porosity parameter $\mathrm{S}$ on $\mathrm{f}^{\wedge^{\prime}}(\eta)$, when $\mathrm{M}=0.0$ and $\beta=1.0$.

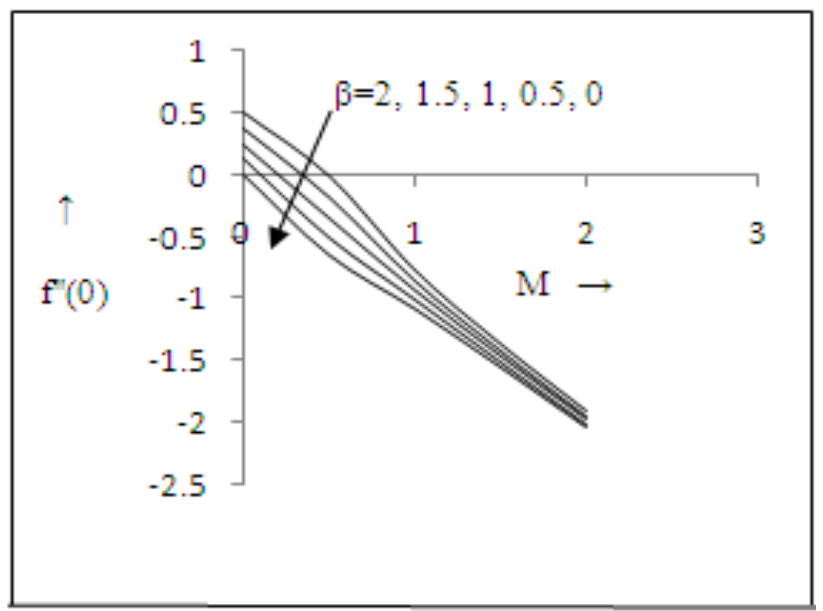

Figure 7: Skin-friction against magnetic parameter $M$ for various values of $\beta$, when $S=0$.

\section{Conclusion}

A MHD boundary layer analysis for an upperconvected Maxwell fluid in a porous medium is presented using He's Homotopy perturbation method (HPM). The governing similarity equation is solved using HPM for various values of the governing parameters to elucidate the salient features of the flow in the presence of these physical parameters. The results have been summarized as the following:

(i) An increase in the strength of the magnetic field results in a decrease in the flow velocity and a corresponding decrease in the thickness of the momentum boundary layer.

(ii) An increase in the Deborah number $\beta$ results in an increase in the flow velocity and thereby increases the thickness of the boundary layer.

(iii) An increase in the porosity parameter $\mathrm{S}$ causes a progressive reduction in the velocity and a corresponding thinning in the boundary layer thickness.

(iv) Skin-friction decreases with an increase in the magnetic field, while the reverse effect is observed for the Deborah number $\beta$.

\section{Acknowledgement}

Support from the Department of Mathematics, Birla Institute of Technology, Jaipur Campus, India is gratefully acknowledged.

\section{Nomenclature}

$B_{0} \quad$ Constant applied magnetic field

$f \quad$ Dimensionless stream function

M Magnetic field parameter 


\section{Chemical Engineering Research Bulletin 18(2015) 12-17}

$\begin{array}{ll}K & \text { Permeability of porous media } \\ S & \text { Porosity parameter } \\ U & \begin{array}{l}\text { Free stream velocity } \\ u, v\end{array} \\ \begin{array}{l}\text { Velocity component of the fluid along the } \mathrm{x} \\ \text { and y directions, respectively }\end{array} \\ \begin{array}{l}\text { Cartesian coordinates along the surface and } \\ \text { normal to it, respectively }\end{array}\end{array}$

Greek symbols

$\begin{array}{ll}\beta & \text { Deborah number } \\ \eta & \text { Dimensionless similarity variable } \\ \rho & \text { Density of the fluid } \\ \mu & \text { Viscosity of the fluid } \\ \nu & \text { Kinematic viscosity } \\ \Psi & \text { Stream function } \\ \lambda & \text { Retardation time } \\ \sigma_{e} & \text { Electrical conductivity }\end{array}$

Superscript

Derivative with respect to $\eta$

Subscripts

$\infty \quad$ Free stream condition

\section{References}

1. Gurion, B., (1984): Single- and multi-phase flows in an electromagnetic field: energy, metallurgical, and solar applications. 4th Edition. American Institute of Aeronautics and Astronautics.

2. Sarpakaya, T., (1961): Flow of non-Newtonian fluids in a magnetic field. AIChE Journal, vol. 7, pp. 324-328.

3. Renardy, M., (1997): High Weissenberg number boundary layers for the upper converted Maxwell fluid. Journal of Non-Newtonian Fluid Mechanics, vol. 68 , no. 1 , pp. $125-132$.

4. Rao, I. J. and Rajagopal, K. R., (2007): On a new interpretation of the classical Maxwell model.Mechanics Research Communications, vol. 34, no. 7-8, pp. 509-514.

5. Qi, H and $\mathrm{Xu}$, M., (2007): Unsteady flow of viscoelastic fluid with fractional Maxwell model in a channel. Mechanics Research Communications, 34(2), pp. 210-212.

6. Hayat, T. and Sajid, M., (2007): Homotopy analysis of MHD boundary layer flow of an upperconvected Maxwell fluid. International Journal of Engineering Science. 45, pp. 393-401.

7. Hayat, T., Fetecau, C., Abbas, Z. and Ali, N. , (2008): Flow of a Maxwell fluid between two side walls due to a suddenly moved plate. Nonlinear Analysis: Real World Applications, 9(5), pp. 2288-2295.
8. Jamil, M., Rauf, A. Zafar, A. A. and Khan, N. A. , (2011): New exact analytical solutions for Stokes' first problem of Maxwell fluid with fractional derivative approach. Computers and Mathematics with Applications. 62(3), pp. 1013-1023.

9. Salah, F., Aziz, Z. A. and Ching, D. L. C., (2011): New exact solution for Rayleigh-Stokes problem of Maxwell fluid in a porous medium and rotating frame. Results in Physics, 1(1), pp. 9-12.

10. Hayat, T., Sajjad, R., Abbas, Z., Sajid, M. and Awatif, A. H., (2011): Radiation effects on MHD flow of Maxwell fluid in a channel with porous medium. International Journal of Heat and Mass Transfer, 54, pp. 854-862.

11. Wang, S. and Tan, W. C., (2011): Stability Analysis of Soret-Driven Double-Diffusive Convection of Maxwell Fluid in a Porous Medium. International Journal of Heat and Fluid Flow, Vol.32, pp. 88-94.

12. Zierep, J. and Fetecau, C., (2007): Energetic Balance for the Rayleigh-Stokes Problem of a Maxwell Fluid. International Journal of Engineering Science, Vol.45, pp. 617-627.

13. Sadeghy, K., Hajibeygi, H. and Taghavi, S. M., (2006): Stagnation-Point Flow of UpperConvected Maxwell Fluids. International Journal of Non-Linear Mechanics, Vol.41, pp. 1242-1247.

14. He, J. H., (1998): Approximate analytical solution for seepage flow with fractional derivatives in porous media. J. Comput. Math. Appl.Mech. Eng, 167 , pp. 57-68.

15. He, J. H., (2000): A review on some new recently developed nonlinear analytical techniques. Int $\mathbf{J}$ Non-linear Sci Numer Simul, 1, pp. 51-70.

16. He, J. H., (2001): Modified Lindstedt-Poincare methods for some non-linear oscillations. Part III: double series expansion. Int. J. Nonlinear Sci Numer Simul, 2, pp. 317-320.

17. He, J.H., (2005): Homotopy perturbation method for bifurcation on nonlinear problems. Int. J. Nonlinear Sci. Numer. Simul, 6, pp. 207-208.

18. He, J. H., (2009): An elementary introduction to the homotopy perturbation method. Computers \& Mathematics with Applications. Volume 57, Issue 3, pp. 410-412.

19. Ariel, P.D., Hayat, T. and Asghar, S., (2006): Homotopy perturbation method and axisymmetric Flow over a stretching sheet. Internat J. Nonlinear Sci. Numer. Simul, 7(4), pp. 399-406.

20. Beléndez, A., Beléndez, T., Márquez, A. and Neipp, C., (2008): Application of He's homotopy perturbation method to conservative truly nonlinear oscillators, Chaos.Solitons \& Fractals, 37(3), pp. 770-780.

21. Ganji, D. D. and Rajabi, A., (2006): Assessment of homotopy-perturbation and perturbation methods 


\section{Chemical Engineering Research Bulletin 18(2015) 12-17}

in heat radiation equations. International Comm. Heat Mass Transfer, 33, pp. 391-400.

22. Ganji, Z. Z. and Ganji, D. D., (2008): Approximate Solutions of Thermal Boundarylayer Problems in a Semi-infinite Flat Plate by using He's Homotopy Perturbation Method. International Journal of Nonlinear Sciences and Numerical Simulation, 9(4), pp. 415-422.

23. Hosein Nia, S. H., Ranjbar, A. N., Ganji, D. D., Soltani, H. and Ghasemi, J., (2008): Maintaining the stability of nonlinear differential equations by the enhancement of HPM. Physics Letters A, 372(16), pp. 2855-2861.

24. Jhankal, A. K., (2014): Homotopy Perturbation Method for MHD Boundary Layer Flow with Low Pressure Gradient over a Flat Plate. Journal of Applied Fluid Mechanics, Vol. 7, No. 1, pp. 177185.

25. Ma, X., Wei, L. and Guo, Z., (2008): He's homotopy perturbation method to periodic solutions of nonlinear Jerk equations. Journal of Sound and Vibration, 314, pp. 217-227.

26. Siddiqui, A.M., Zeb, A., Ghori, Q. K. and Benharbit, A. M., (2008): Homotopy perturbation method for heat transfer flow of a third grade fluid between parallel plates, Chaos, Solitons \& Fractals, 36(1), pp. 182-192.
27. Zhang, L. N. and He, J. H., (2006): Homotopy perturbation method for the solution of the electrostatic potential differential equation, Mathematical Problems in Engineering, Art. No. 83878 .

28. Zhang, B. G., Li, S. Y. and Liu, Z. R., (2008): Homotopy perturbation method for modified Camassa-Holm and Degasperis-Procesi. Physics Letters A, 372(11), pp. 1867-1872.

29. Hosein Nia, S. H., Ranjbar, A. N., Ganji, D. D., Soltani, H. and Ghasemi, J., (2008): Maintaining the stability of nonlinear differential equations by the enhancement of HPM. Physics Letters A, 372(16), pp. 2855-2861.

30. Bansal, J. L., (1977): Viscous Fluid Dynamics, Oxford \& IBH Pub. Co., New Delhi, India.

31. Bansal, J. L., (1994): Magnetofluiddynamics of Viscous Fluids, Jaipur Publishing House, Jaipur, India.

\section{Available online at http://www.banglajol.info/index.php/CERB}

Publisher: Department of Chemical Engineering, Bangladesh University of Engineering and Technology (BUET). Review \&Publication: A submitted original manuscript is taken into review only if the uniqueness is found to be more than $85 \%$ in plag-scanning and selected for publication by the complete acceptance from at least two reviewers out of three. Home Page: http://www.banglajol.info/index.php/CERB . Indexed by Chemical Abstract Service (CAS), CEABA-VtB, Google Scholar, Scopus and DOAJ. 